\title{
Instabilities in Droplets Spreading on Gels
}

\author{
Karen E. Daniels, ${ }^{1,2}$ Shomeek Mukhopadhyay, ${ }^{1}$ Paul J. Houseworth, ${ }^{2}$ and Robert P. Behringer ${ }^{1}$ \\ ${ }^{1}$ Department of Physics and Center for Nonlinear and Complex Systems, Duke University, Durham, North Carolina 27708, USA \\ ${ }^{2}$ Department of Physics, North Carolina State University, Raleigh, North Carolina, 27695, USA \\ (Received 31 July 2006; revised manuscript received 28 February 2007; published 18 September 2007)
}

We report a novel surface-tension driven instability observed for droplets spreading on a compliant substrate. When a droplet is released on the surface of an agar gel, it forms arms or cracks when the ratio of surface-tension gradient to gel strength is sufficiently large. We explore a range of gel strengths and droplet surface tensions and find that the onset of the instability and the number of arms depend on the ratio of surface tension to gel strength. However, the arm length grows with an apparently universal law $L \propto t^{3 / 4}$.

The surface-tension driven spreading of liquids is industrially and biologically important, and has been studied in detail on both solid and liquid substrates [1-3]. Less is known about how droplet spreading is modified in the presence of a compliant substrate, a situation especially relevant to biological applications $[4,5]$. We perform dropletspreading experiments on gel agar, a viscoelastic material, to explore the influence of substrate on the spreading dynamics of the droplet. We find a novel branching instability with an onset that is controlled by the ratio of surfacetension difference to the shear strength of the gel. The existence of a spreading morphology in which a spreading droplet becomes spatially localized has important implications for the industrial and medical application of surfactants.

Droplets spread differently on liquids, which are mobile, than on solids, which are essentially rigid $[3,6,7]$. The present experiments on spreading on a viscoelastic substrate (gel agar) are intended as a way to span these two limits: by increasing the agar concentration, one can tune the substrate from liquidlike to solidlike behavior. The difference in surface tension between the droplet [polydimethylsiloxane (PDMS), a silicone oil; or octylphenoxy polyethoxy ethanol (Triton X-305 from Dow Chemical), a surfactant solution] and the substrate drives the spreading process.

Previous work on the spreading of droplets on gel substrates $[8,9]$ showed circular spreading with rates intermediate between those observed on solid and liquid substrates, contrary to what we find. In addition, prior studies of viscoelastic substrates have focused on substrate breakup or fracture when subjected to stresses [10-13]. Here, we observe a failure of the gel driven by surface tension. After the initial failure, there are two morphologically different manifestations of the instability, influenced by both the substrate elasticity and the surface tensions, which we call starbursts and wispy drops. For weak gels (shear modulus $G \lesssim 30 \mathrm{~Pa}$ ), the drop breaks into distinct cracklike spreading arms in a starburst formation, as shown in Fig. 1(a). Morphologically, this is similar to cracking patterns observed in $[10,12]$. We observe that the arms have steep sides extending into the gel and are filled with material from the spreading droplet. Above the onset of the starburst instability, the rate of spreading is found to be controlled only by the width of the arms, with collapse in the data across substrate modulus, surface tension, viscosity, and droplet volume. Well above the gelation transition $(G \gtrsim 30 \mathrm{~Pa})$, the drops are typically circular as on a prewet solid substrate; i.e., there is no indication of failure. However, after long times some droplets (on gels near $G \approx 30 \mathrm{~Pa}$ ) demonstrate the wispy morphology shown in Fig. 1(b). We investigate the morphology and dynamics of the starbursts in terms of droplet surface tension, viscosity, and volume, and the strength of the gel.
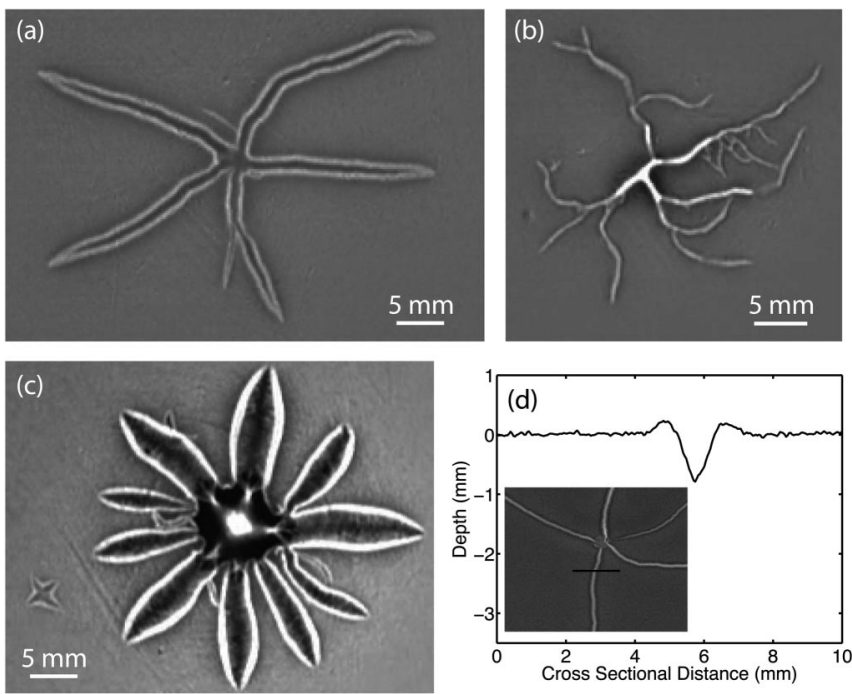

FIG. 1. Sample images of droplets spreading on gel agar substrates. (a) -(c) Shadowgraph images adapted from [16]; bright lines represent the outer boundaries of the arms. (a) Triton X-305 solution starburst: droplet concentration $\phi=$ $50 \mathrm{ppm}(\sigma=63 \mathrm{mN} / \mathrm{m}), X=0.12 \% \mathrm{w}(G=16 \mathrm{~Pa})$, and $V=$ $5 \mu \mathrm{L}$ after $14 \mathrm{~s}$. (b) Triton X-305 solution wispy drop: droplet concentration $\phi=100 \mathrm{ppm}(\sigma=60 \mathrm{mN} / \mathrm{m}), X=0.14 \% \mathrm{w}$ $(G=28 \mathrm{~Pa}$ ), and $V=5 \mu \mathrm{L}$ after 27 s. (c) PDMS starburst: $\sigma=20 \mathrm{mN} / \mathrm{m}, X=0.08 \% \mathrm{w}(G=1.3 \mathrm{~Pa}), \nu=1000 \mathrm{cS}$, and $V=5 \mu \mathrm{L}$ after $5 \mathrm{~s}$. (d) Scattering image of Triton X-305 starburst (inset) and cross section of arm. 
The substrate is made from agar, a polymer composed of subunits of galactose (a sugar); agar undergoes a physical gel transition for weight concentrations of agar above $0.014 \%$ weight in water at $20.0{ }^{\circ} \mathrm{C}$ [14]. We express agar concentrations as $X=M_{\text {agar }} /\left(M_{\text {agar }}+M_{\text {water }}\right) \%$ w, where $M$ is the mass of each component of the gel. We examine droplets spreading on $\approx 5 \mathrm{~mm}$ thick gels with concentrations $0.06 \leq X \leq 0.14$. These values correspond to a shear modulus of $1 \mathrm{~Pa} \lesssim G \lesssim 30 \mathrm{~Pa}$ [14] and a surface tension $67 \mathrm{mN} / \mathrm{m} \lesssim \sigma \lesssim 75 \mathrm{mN} / \mathrm{m}$. We measure the latter using the pendant drop method [15]. A full treatment of this problem would involve a correction for the gel elasticity; however, this correction is small except for our stiffest gels. For $X \leqq 0.06 \%$ w the droplets spread out as on water, and for $X \geqslant 0.014 \%$ w they remain circular and small $(\mathrm{mm}$ radius) [16].

We use two types of droplets, Triton X-305 at concentrations $\phi=1$ to $1000 \mathrm{ppm}$ in deionized water and pure PDMS at several viscosities and surface tensions. Triton $\mathrm{X}-305$ is a nonionic surfactant with molecular weight $\approx 1526$; its critical micellar concentration is $2000 \mathrm{ppm}$ at $23^{\circ} \mathrm{C}$ [17]. The kinematic viscosity $\nu$ of the Triton X-305 solution is approximately that of pure water, $0.89 \mathrm{cS}$, and its surface tension $\sigma_{d}$ ranges from that of distilled water $(73 \mathrm{mN} / \mathrm{m})$ at the lowest $\phi$ down to $50 \mathrm{mN} / \mathrm{m}$ at $\phi=$ 1000 [17]. PDMS has a density $\rho=0.968 \mathrm{~g} / \mathrm{cm}^{3}$ and the samples used had surface tensions $\sigma_{d}=2.25$ to $20.0 \mathrm{mN} / \mathrm{m}$ and shear viscosities $\nu=10$ to $1000 \mathrm{cS}$.

We visualize the spreading droplets using shadowgraphy [18] through gel substrates prepared in clear plastic petri dishes. A parallel beam of light passes through the material from below; an image is formed on a ground glass above the layer and captured by a digital camera and framegrabber. In addition, to visualize the arm topography we extract a calibrated cross section using light scattering in gels containing $1 \mu \mathrm{m}$ polystyrene spheres [19]. Each droplet is ejected from a micropipette held about $1 \mathrm{~cm}$ above the surface of the gel. We examined droplets with volume $V=$ 0.1 to $10 \mu \mathrm{L}$. For reference, a $V=5 \mu \mathrm{L}$ of fluid with a molecular size of $10^{-8} \mathrm{~m}$ (PDMS), corresponds to a monolayer surface film with radius $\approx 12 \mathrm{~cm}$.

For the starbursts, the key physics involves a competition between spreading due to surface tension and the gel rigidity, ultimately resulting in the fracture of the gel substrate. A droplet on a gel surface will spread if it has a spreading parameter $\sigma_{g}-\sigma_{d}-\sigma_{g d}>0$, for gel-air, droplet-air, and gel-droplet surface tensions, respectively. Since both the gel and the Triton X-305 droplets are largely composed of water, we begin by assuming that $\sigma_{g d}$ is negligible and define $\Delta \sigma \equiv \sigma_{g}-\sigma_{d}$; below we will examine the consequences of this assumption for the PDMS droplets. The forces that drive the radial spreading of the drop deform the gel substrate, as shown schematically in the inset to Fig. 2, and we estimate the energies involved. Suppose that there is a nascent crack of width $d$ (corresponding roughly to the length of a stretched polymer at the point of failure) in the radial direction, and depth $h$ into the gel. This crack extends in the plane of the gel over some fraction of the outer circumference of the drop, $a R$. There are now several relevant energies: the energy associated with the surface tension stretching $E_{\sigma} \sim \Delta \sigma a R d$, the elastic energy at fracture $E_{e} \sim G a R d h$, and the thermal energy $E_{T}=k_{B} T$. Here, we assume that the failure modulus of the gel is proportional to the elastic modulus $G$. The time to nucleate a crack, $\tau_{\text {nuc }} \sim \exp \left(-E_{b} / E_{T}\right)$, involves an energy barrier depending on $E_{e}, E_{\sigma}$, and $E_{T}$. Assuming that $d$ is comparable in size to an extended gel polymer strand, and that $h$ is also microscopically small, the first two energies are both many orders of magnitude larger than $E_{T}$. Consequently, we expect (and observe) rapid nucleation. During $\tau_{\text {nuc }}$, fluid from the drop diffuses a distance $\lambda \sim$ $\left(D \tau_{\text {nuc }}\right)^{1 / 2}$ into the gel. $\lambda$ then sets the depth for the crack: $h=\lambda$. Therefore, we might expect a Marangoni or elastic instability above a critical value of

$$
M \equiv \frac{\Delta \sigma}{\lambda G}=\frac{E_{\sigma}}{E_{e}} .
$$

We characterize the Marangoni/elastic instability in terms of several simple measures: the number of arms that form, their mean width, and their length as a function of time. Figure 2 indicates that the parameter $M$ captures a key feature of the experiments, namely, the number of arms. This number increases with $\Delta \sigma / G$; here we omit the factor $\lambda$, although we expect that it depends on the diffusivity of the droplet in the gel. However, data for such diffusivities are not available and are likely viscosity dependent for the PDMS droplets. The number of arms increases for decreasing $G$ (connect identical symbols, best seen for the solid stars and solid line), or for decreasing $\sigma_{d}$ (for example, dashed lines connecting open symbols of the same color). In the former case, onset occurs by changing the gel concentration for a constant PDMS droplet, and in the later by changing the surfactant concentration of the Triton X-305 droplet for a constant gel. There is scatter in the plot due to a number of uncontrolled factors, for instance heterogeneities in the gel and the impact velocity of the droplet. Remarkably, in spite of the 3 orders of magnitude difference in viscosity for the two types of droplets, and the neglect of the $\sigma_{g d}$ term for PDMS, we achieve a reasonable collapse of Figs. 2(a) and 2(b) by assuming $\lambda_{\mathrm{PDMS}}=12 \lambda_{\text {Triton }}$. Ignoring viscosity dependence, this suggests that PDMS diffuses more slowly than Triton X-305, in accordance with its longer molecular length. An alternative framework in which to view the instability is to consider that the number of arms in the Marangoni/elastic instability decreases with increased surface tension of the droplet. This is to be expected since the wavelength of the instability increases with surface tension in general for interfacial phenomena [20].

Several limits of Eq. (1) are instructive. For droplets with sufficiently high surface tension (low concentration of Triton X-305, low $\Delta \sigma$ ), the central droplet does not spread significantly and the gel remains intact, resulting in no arm 


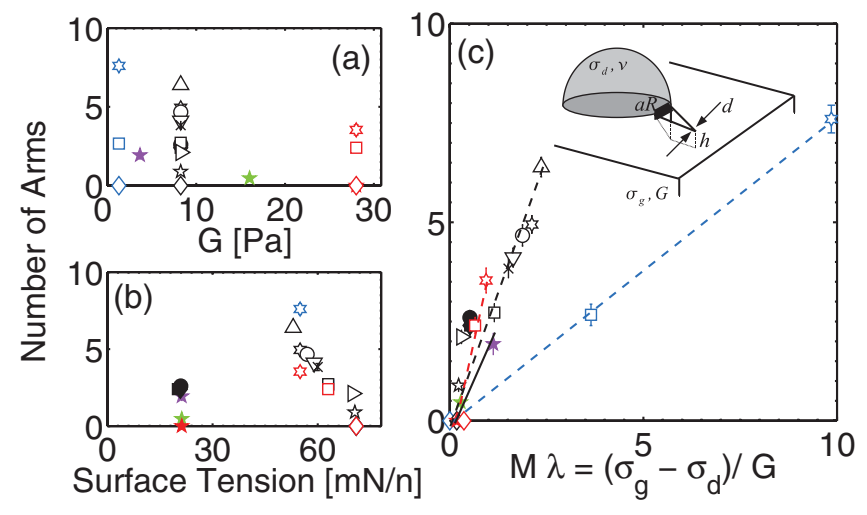

FIG. 2 (color). Mean number of arms on individual droplets as a function of (a) droplet surface tension $\sigma_{d}$; (b) gel elastic modulus $G$; and (c) $M \lambda$ [see Eq. (1)], assuming $\lambda_{\text {PDMS }}=$ $12 \lambda_{\text {Triton. }}$ Inset: Schematic of Marangoni/elastic instability. Open symbols are Triton X-305 solutions, with $\sigma_{d}=$ $72 \mathrm{mN} / \mathrm{m}(\triangleleft), 71 \mathrm{mN} / \mathrm{m}(\diamond), 70.6 \mathrm{mN} / \mathrm{m}(\star), 70 \mathrm{mN} / \mathrm{m}$ $(\triangleright), 63 \mathrm{mN} / \mathrm{m}(\square), 60 \mathrm{mN} / \mathrm{m}(\times), 59 \mathrm{mN} / \mathrm{m}(\nabla), 57 \mathrm{mN} / \mathrm{m}$ (○), $55 \mathrm{mN} / \mathrm{m}(*), 53 \mathrm{mN} / \mathrm{m}(\triangle), 50 \mathrm{mN} / \mathrm{m}(+)$. Solid symbols (here overlapping) are PDMS: $\sigma_{d}=20.1 \mathrm{mN} / \mathrm{m}$ and $\nu=$ $10 \mathrm{cS}(\boldsymbol{\square}), \quad \sigma_{d}=20.8 \mathrm{mN} / \mathrm{m}$ and $\nu=50 \mathrm{cS}(\bullet), \sigma_{d}=$ $20.9 \mathrm{mN} / \mathrm{m}$ and $\nu=100 \mathrm{cS}(\boldsymbol{O}), \sigma_{d}=21.2 \mathrm{mN} / \mathrm{m}$ and $\nu=$ $1000 \mathrm{cS}(\star)$. Symbol colors represent gel substrate: $1.3 \mathrm{~Pa}$ (blue), 3.7 Pa (magenta), 5.6 Pa (cyan), 8.2 Pa (black), 11.7 Pa (yellow), $16 \mathrm{~Pa}$ (green), $28 \mathrm{~Pa}$ (red). Droplet volume is $2.5 \mu \mathrm{L}$. Each point is averaged over at least ten drops at the same parameters. Horizontal error bars are due to $G$ and $\sigma_{g}$; vertical bars are the standard error for the measurements. No data at $\phi=$ $1000 \mathrm{ppm}$ are included, due to the unreliable counting of numerous arms.

growth. Similarly no arms appear for gels with $G \gtrsim 70 \mathrm{~Pa}$; instead, the droplets spread as on a solid [2,21]. For $30 \mathrm{~Pa} \lesssim G \lesssim 70 \mathrm{~Pa}$, a different morphology involving the growth of thin, branching wisps was observed for Triton X-305 droplets (but not PDMS), as shown in Fig. 1(b) and [16]. Conversely, for gels with $G \lesssim 1$, the substrate is essentially a viscous fluid and the droplet spreads out rapidly, as on a deep liquid layer. In Fig. 2, note that the open blue symbols (performed on gels with $G=1.3 \mathrm{~Pa}$ ) have a consistently low number of arms, suggesting that the mechanism is nearing its limit of applicability: the gel no longer has a yield strength. For the remainder of this work, we will focus on the starbursts.

For each of the major arms in a starburst pattern, we track the length $L(t)$ as a function of the time from its initiation, as shown in Fig. 3. After $\sim 10 \mathrm{~s}$, the evolution of the pattern is substantially accomplished, and the scaling discussed below typically breaks down. For each arm, we fit $L(t)$ to $L=A t^{\alpha}$. Figure 4 shows that the fit value of the exponent $\alpha$ is consistently close to $3 / 4$, independent of substrate gel modulus, droplet surface tension, arm width, and volume. In addition, the factor of 1000 in viscosity does not appear to affect $\alpha$, which is indistinguishable for the PDMS and Triton X-305 data.

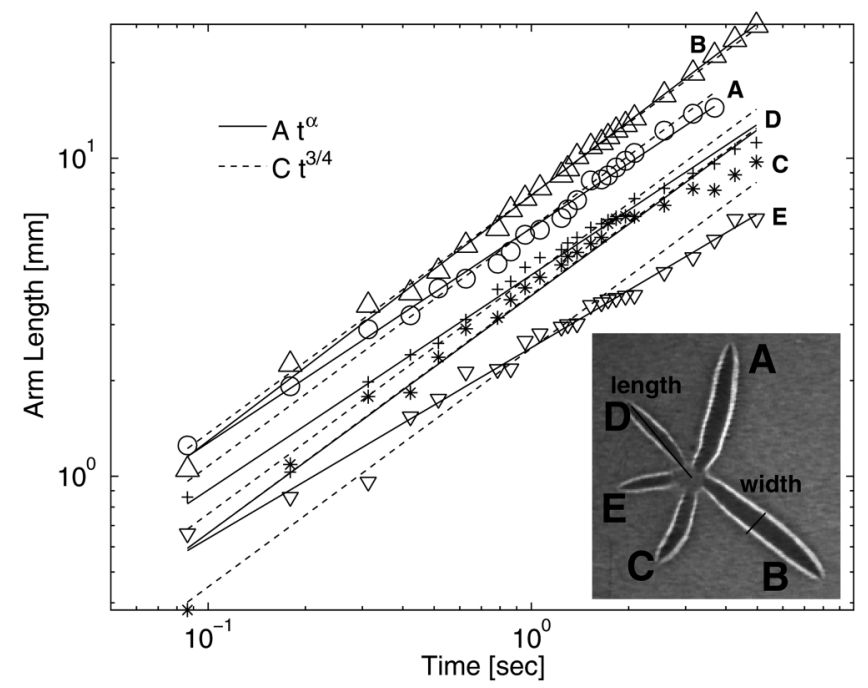

FIG. 3. Length of the five arms (A, B, C, D, E) of a Triton X305 starburst (inset) at a function of time. Time $t$ is measured from the start of each arm's growth, not the drop release time. Solid lines are fits with a free exponent; dashed lines are fixed $\alpha=3 / 4$. Gel is $3.7 \mathrm{~Pa}$ and droplet has $V=5 \mu \mathrm{L}$ and $\sigma_{d}=$ $60 \mathrm{mN} / \mathrm{m}$.

This time dependence is the same as for oil spreading axisymmetrically on water [3], where the value of the exponent arises due to the competing effects of viscous drag and surface-tension forces. In the liquid-on-liquid case, the prefactor

$$
C \propto(\Delta \sigma)^{1 / 2}(\rho \mu)^{-1 / 4}
$$

has a weak dependence on the viscosity $\mu$ (as well as
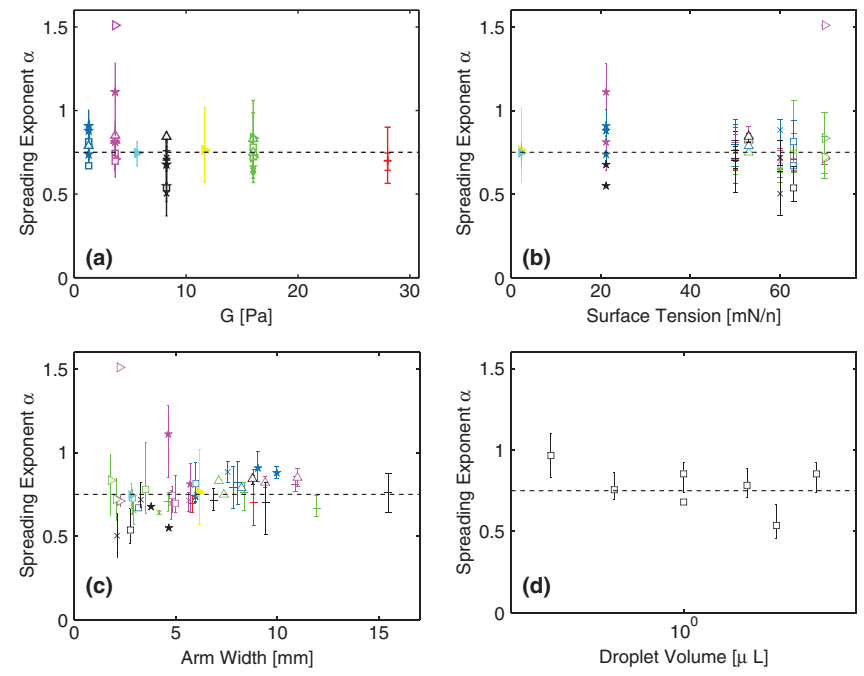

FIG. 4 (color). Fit value of spreading exponent $\alpha$ vs (a) shear modulus $G$, for constant $V=5 \mu \mathrm{L}$, (b) surface tension $\sigma_{d}$, for constant $V=5 \mu \mathrm{L}$, (c) arm width, for constant $V=5 \mu \mathrm{L}$, and (d) droplet volume, $V$, for constant gel and droplet properties. Points are averaged over the arms of a single droplet. Legend as given in Fig. 2. 


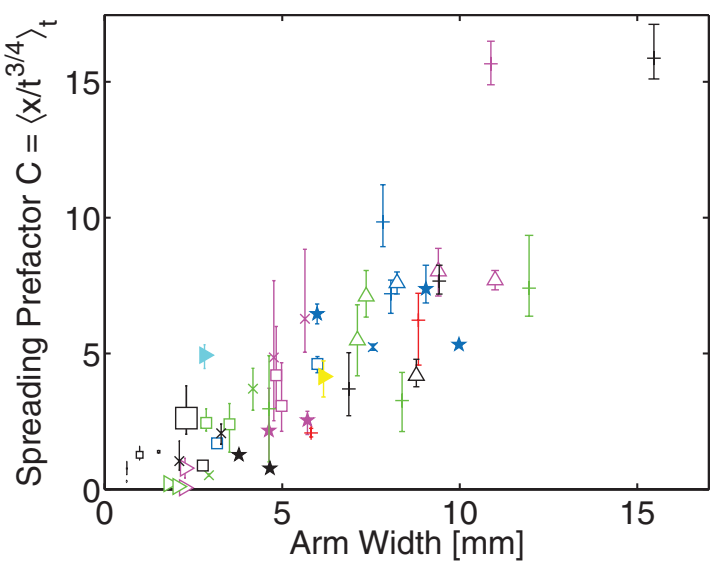

FIG. 5 (color). Mean spreading prefactor $C$, in $\mathrm{mm} / \mathrm{sec}^{3 / 4}$, as a function of mean arm width. Each point is averaged over the arms of a single drop, with max or min values shown as vertical bars. See the legend in Fig. 2 for parameters of each point. Size of symbol indicates droplet size, with the majority taken at $V=5 \mu \mathrm{L}$.

density $\rho$, which we did not vary significantly in our experiments). Intriguingly, recent work on the surfactantinduced fracture of a particle raft [12], saw a multiarmed morphology similar to the starbursts observed here and $t^{3 / 4}$ spreading behavior, which the authors associated with liquid-on-liquid behavior.

In order to obtain more precise values of $C$, we assume that $\alpha=3 / 4$ is universally applicable. We then determine the time-averaged value of the prefactor using $C=$ $\left\langle L(t) / t^{3 / 4}\right\rangle_{t}$; this parameter sets the scale of the spreading rate. Its value is approximately the same for both PDMS and Triton X-305 droplets in spite of the difference in viscosity, echoing Eq. (2). Although there does not appear to be any significant dependence of $C$ on viscosity, the average value of $C$ is linearly related to the average arm width for a given droplet. We note that the arm width at half-length increases and/or decreases somewhat during arm growth, again likely due to heterogeneities in the gel. Since the variation in width over the course of the growth is small compared to the length of the arms, we consider its time-averaged value. As shown in Fig. 5, the wider the arms of a droplet, the faster they grow (larger $C$ ). Remarkably, data for the various gel moduli, droplet volumes, surface tensions, and droplet material (PDMS vs Triton X-305) are all consistent with this single linear relationship.

In addition to instabilities due to tangential stresses described in Eq. (1), normal stresses due to gravity may be relevant as well. We consider the ratio

$$
\frac{\rho V g}{(\Delta \sigma) R}
$$

and find a value of about 5, indicating that the gravitational and surface-tension effects are similar in magnitude.
Although the number of starburst arms slowly increased with droplet volume, we observed starbursts for the smallest volumes that we could generate [see small starburst at left side of Fig. 1(c)].

A key issue is the nature of the mechanism through which the arms advance. The initially smooth gel cracks under the tangential stress from the surface-tension gradient imposed by the droplet. Once this process has begun, the crack opens further due to both the curvature at the tip and surface-tension forces due to that curvature. The tip velocity is set by the viscous spreading of the droplet fluid into the opening crack, with resulting dynamics similar to that of the liquid-on-liquid case [Eq. (2)]. Time-resolved measurements of the initial stages of starburst formation will be necessary to further clarify these issues. The novelty of this cracking instability is that it is driven by an advancing layer of fluid.

This work has been supported by NSF Grant No. DMS0244498 and by North Carolina State University. We thank the UCLA/Duke/NCSU Thin Films Group, Bruno Andreotti, and L. Mahadevan for helpful conversations.

[1] S. M. Troian, X. L. Wu, and S. A. Safran, Phys. Rev. Lett. 62, 1496 (1989).

[2] M. Cachile et al., Colloids Surf. A 159, 47 (1999).

[3] D. P. Hoult, Annu. Rev. Fluid Mech. 4, 341 (1972).

[4] K. Khanvilkar, M. D. Donovan, and D. R. Flanagan, Adv. Drug Delivery Rev. 48, 173 (2001).

[5] D. Halpern, O. E. Jensen, and J. B. Grotberg, J. Appl. Physiol. 85, 333 (1998).

[6] J. Lopez, C. A. Miller, and E. Ruckenstein, J. Colloid Interface Sci. 56, 460 (1976).

[7] L. H. Tanner, J. Phys. D 12, 1473 (1979).

[8] D. Szabo et al., J. Chem. Phys. 1138253 (2000).

[9] D. Kaneko et al., J. Polym. Sci., B Polym. Phys. 43, 562 (2005).

[10] A. Levermann and I. Procaccia, Phys. Rev. Lett. 89, 234501 (2002).

[11] E. R. Dufresne et al., Phys. Rev. Lett. 91, 224501 (2003).

[12] D. Vella et al., Phys. Rev. Lett. 96, 178301 (2006).

[13] D. Bonn et al., Science 280, 265 (1998).

[14] M. Tokita and K. Hikichi, Phys. Rev. A 35, 4329 (1987).

[15] J. M. Andreas, E. A. Hauser, and W. B. Tucker, J. Phys. Chem. 42, 1001 (1938).

[16] K.E. Daniels, S. Mukhopadhyay, and R.P. Behringer, Chaos 15, 041107 (2005).

[17] J. Zhang and R. M. Manglik, J. Heat Transfer 127, 684 (2005).

[18] G.S. Settles, Schlieren and Shadowgraph Techniques: Visualizing Phenomena in Transparent Media (Springer, New York, 2001).

[19] W. B. Wright, R. Budakian, and S. J. Putterman, Phys. Rev. Lett. 76, 4528 (1996).

[20] W. W. Mullins and R. F. Sekerka, J. Appl. Phys. 35, 444 (1964).

[21] M. Cachile et al., Physica (Amsterdam) 329A, 7 (2003). 